\title{
Lock and Key Hypothesis - A New Perspective
}

Apar Gupta

Founder, Blu Ocean Studios Private Limited, India.

DOI: http://doi.org/10.38177/ajast.2021.5303

Copyright: () 2021 Apar Gupta. This is an open access article distributed under the terms of the Creative Commons Attribution License, which permits unrestricted use, distribution, and reproduction in any medium, provided the original author and source are credited.

Blu Ocean Studios Private Limited is an organization, which has been founded under the firm belief of using research and innovation to drive authentic solutions in the field of Healthcare and wellness. The world has been facing a larger turmoil either due to the environmental factors, the epidemic and other reasons. We have witnessed a complete lockdown on the planet because of a virus, that caused a menace and fight is still on. Probably the next virus and similar attack might not give a chance to humans to prepare a vaccine or an antidote. Henceforth, we believe that there should be a focus on what we call as a Lock and Key Hypothesis wherein focus should be to explore and support new Science and Technologies rather on what has been done by our predecessors or other available technologies.

Keywords: Key, Hypothesis, Ecosystem, Perspective, Invention, Blu ocean, Healthcare.

Blu Ocean Studios Private Limited is an organization, which has been founded under the firm belief of using research and innovation to drive authentic solutions in the field of Healthcare and wellness. The company aims to solve perennial problems of healthcare through some authentic scientific innovations.

My name is Apar Gupta, founder at Blu Ocean Studios Pvt Ltd, an ardent believer in the power of Science and Technology. The world has been facing a larger turmoil either due to the environmental factors, the epidemic and other reasons. We have witnessed a complete lockdown on the planet because of a virus, that caused a menace and fight is still on. But I believe that such an event also poses a further threat as whether we are prepared for such events, probably the next virus and similar attack might not give a chance to humans to prepare a vaccine or an antidote. Henceforth, we believe that there should be focus on what we call as a Lock and Key Hypothesis wherein focus should be to explore and support new Science and Technologies rather on what has been done by our predecessors or other available technologies. There exists a drying research pipeline, as we can witness that even the most accurate science such as Vaccines are not offering a complete solution and protection towards the attack of Covid virus, as people have died even after vaccination. This is yet another strong example that new areas of science need to be explored.

The lock and key hypothesis emphasizes that when a problem is unique and novel, we should try to explore unique and novel solutions, but instead, majority of people still try to explore solutions within existing technologies, rather exploring or creating authentic and new technologies. Henceforth, with a new lock, you need a new key, rather using the existing key which eventually turns out waste or has been compromised with over exploitation and excessive usage.

It's time to indulge ourselves in designing authentic yet powerful and complete solutions to some of the biggest problems, keep on innovating and experimenting using the best modern day technologies to counter other problems and provide newer and sustainable solutions. Our aim and emphasis should lie on groundbreaking research and authentic technologies that are able to meet the challenges of today, primarily in the field of healthcare. 


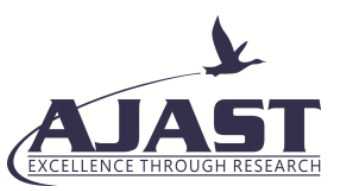

Asian Journal of Applied Science and Technology (AJAST)

Volume 5, Issue 3, Pages 32-34, July-September 2021

Its time that the relevant stakeholders such as the Startup Ecosystem, the healthcare NGO's and similar institutions should support companies who doesn't speak for the politics of greed or themselves but for the entire humanity.

Man landed on the moon in 1960's but hasn't been able to go beyond and step the foot, it's because we are still believing and using the technologies that were best fit to that problem, but they are no longer sustainable when the problem has changed.

Different problems require solutions that are curated in the specifications of that problem. For example, we can look at the curious story of Alan Turing. As the Germans used Enigma coded messages to communicate, the British troops tried decoding the message with the same procedure and practices. The best of minds could not decipher the message as they used the existing techniques. Alan Turing provided them with a fresh perspective to decode the problem, and he managed to crack down the entire mechanism used to code Enigma messages. He could take down an entire communication system because he could use "a new key for a new lock". We pride ourselves on using the approach which helps us deal with the problem with renewed enthusiasm. The most difficult "puzzles" can be solved when you look at them with different and unique perspective.

It is evident from the current situation that a major portion of the healthcare industry is focused on reusing the same formulas to manufacture medicines for different diseases. Hence most of the time, they find themselves at cul-da-sac of inventions. We want to break this chain and make way for new inventions.

In any field, to foster new inventions, we need to make way for new ideas. We must take on a new approach while solving any problem. It is normal to use previously applied solutions. But all this will just help in reducing the investment. We suggest taking a fresh approach to tackle problems that might require a specific curated approach. After all, "reference" and "copying" are two different words with completely different meanings and people are trying to mix both, when they actually trying to copy, rather referring.

Blu Ocean Studios Private Limited is a name inspired from 'Blu Ocean Strategy' that emphasizes on designing unique, creating and crafting novel solutions. We are ardent believers in Science and technology, and believe that the universe holds the possible solution to every problem, and authentic and deep technologies does sounds like a fiction until developed. Through this lock and key hypothesis, we aim to emphasize on the point that for newer problems, we require new solutions which can solve the problems completely.

We are a Startup whose primary focus is to improve the healthcare ecosystem through authentic inventions, with our focus to develop a technology that might be able to control any Animal cell, this is also indicative that such science could also provide a solution to one of the most stubborn puzzle, known as Cancer, with other area of applications if it could be explored further.

We believe that thought and vision is the basic to develop any technology

\section{Declarations}

Source of Funding

This work did not receive any grant from funding agencies in the public, commercial, or not-for-profit sectors. 


\section{Competing Interests Statement}

The author declares no competing financial, professional and personal interests.

\section{Ethical Approval}

Ethical approval for this research was based on organizational guidelines.

\section{Consent for publication}

Author declares that he/she consented for the publication of this research work.

\section{Availability of data and material}

Author is willing to share data and material according to the relevant needs.

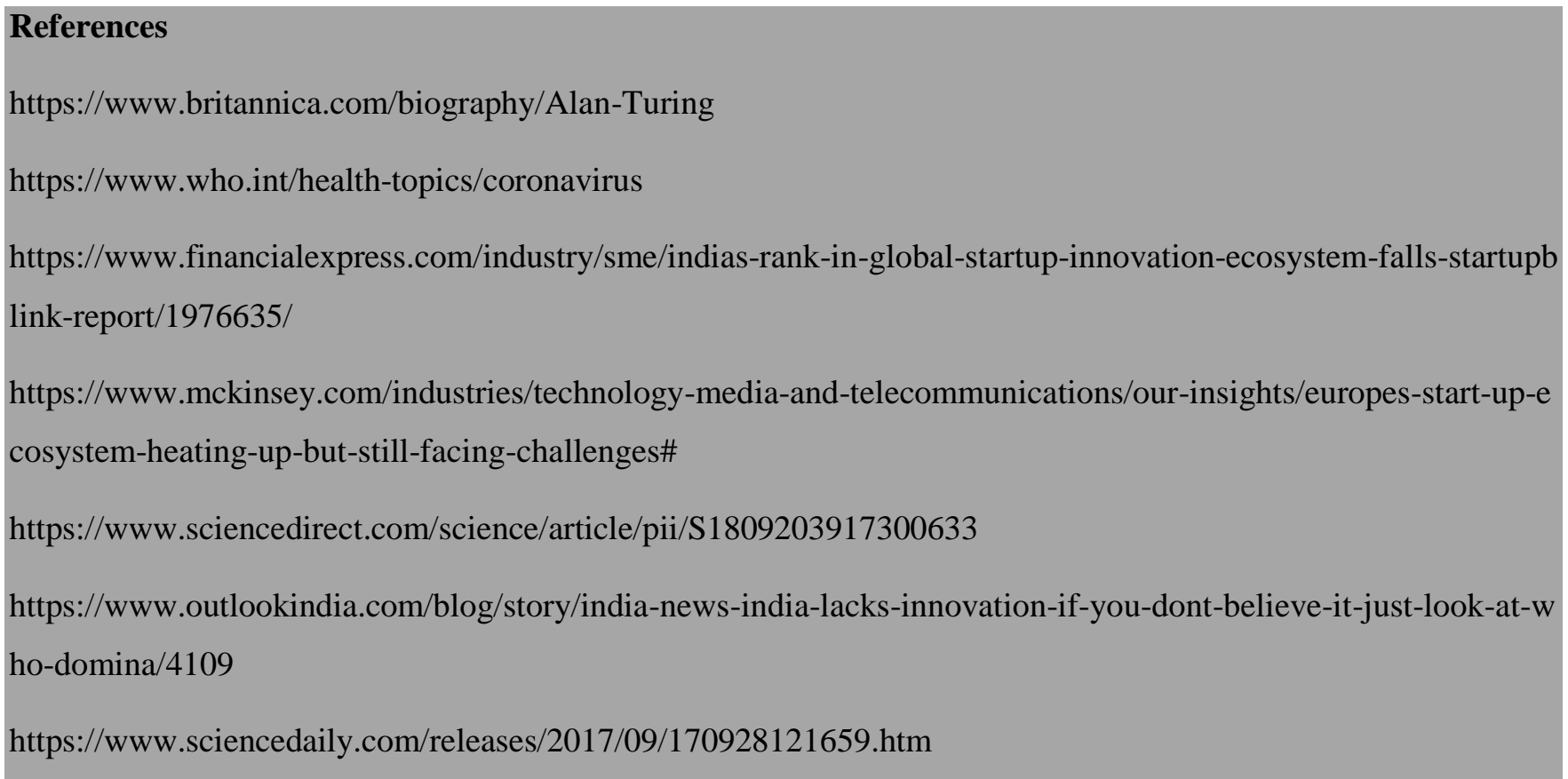

